\title{
A Pattern Similarity Scheme for Medical Image Retrieval
}

\author{
Dimitris K. Iakovidis, Member, IEEE, Nikos Pelekis, Haralampos Karanikas, Evangelos Kotsifakos, \\ Ioannis Kopanakis, and Yannis Theodoridis
}

\begin{abstract}
In this paper we propose a novel scheme for efficient content-based medical image retrieval, formalized according to the PANDA (PAtterns for Next generation Database systems) framework. The proposed scheme involves low-level feature extraction from image regions followed by clustering of the feature space to form higher-level patterns. The components of each pattern include a cluster representation and a measure that quantifies the quality of the image content representation achieved by the pattern. The similarity between two patterns is estimated as a function of the similarity between both the structure and the measure components of the patterns. Experiments were performed on a reference set of radiographic images, using standard wavelet domain image features. The results show that the proposed scheme can be more efficient than the common ground schemes for medical image retrieval, as it does not involve exhaustive, nearest neighbor searching over the whole set of the available feature vectors. Keeping the patterns in a unified form facilitates further processing and analysis by mining or visualization algorithms.
\end{abstract}

\section{INTRODUCTION}

$\mathrm{O}^{\mathrm{n}}$ ne of the primary tools used by physicians is the comparison of previous and current medical images associated with pathologic conditions. As the amount of pictorial information stored in both local and public medical databases is steadily growing, efficient image indexing and retrieval is increasingly becoming a necessity.

In the last decade the advances in information technology allowed the development of Content-Based Image Retrieval (CBIR) systems, capable of retrieving images based on their similarity with one or more query images. Some of these systems are QBIC [1], VisualSEEK [2], Virage [3], Netra [4], PicSOM [5], SIMPLicity [6], CIRES [7], and FIRE [8] . More than fifty CBIR systems are surveyed in [9].

Manuscript received June 30, 2006. This work was partially supported by the MetaOn project co-funded by the Operational Programme "Information Society" of the Greek Ministry of Development, General Secretariat for Research and Technology and by the European Union.

D. K. Iakovidis is with the Knowledge Management Laboratory and with the Department of Informatics and Telecommunications, University of Athens, GR 15784 Panepistimiopolis, Ilisia, Greece (e-mail: dimitris.iakovidis@ieee.org).

N. Pelekis is with the Department of Informatics, University of Piraeus, Greece (email: npelekis@unipi.gr).

H. Karanikas is with the Knowledge Management Laboratory, University of Athens, Greece (email: bkaranikas@phs.uoa.gr)

E. Kotsifakos is with the Department of Informatics, University of Piraeus, Greece (email: ek@unipi.gr).

I. Kopanakis is with the Knowledge Management Laboratory, University of Athens, Greece.

Y. Theodoridis is with the Department of Informatics, University of Piraeus, Greece (email: ytheod@unipi.gr).
The benefits emanating from the application of contentbased approaches to medical image retrieval range from clinical decision support to medical education and research [10]. These benefits motivated researchers either to apply general purpose CBIR systems to medical images [8] or to develop new ones explicitly oriented to specific medical domains. Specialized CBIR systems have been developed to support the retrieval of various kinds of medical images, including High Resolution Computed Tomographic (HRCT) images [11], breast cancer biopsy slides [12], Positron Emission Tomographic (PET) functional images [13], ultrasound images [14], endoscopic images [15], pathology images [16], spine radiographs [17], and mammographic images [18].

Common ground for most of the systems cited above is that image retrieval is based on similarity measures estimated directly from low-level image features, whereas it involves multidimensional, usually exhaustive, nearest neighbor searching over the whole set of the available feature vectors. However, such an approach can be time consuming with large image databases.

Research on improving the efficiency of the image retrieval process has mainly focused on image indexing techniques by utilizing data structures, such as R-trees [1][19], feature index trees [20], iconic index trees [21], and meshes of trees [22]. Other approaches to improving efficiency, include clustering of the image feature spaces [23][24], and utilization of alternative similarity measures, usually dependent on feature sets [25][26].

Motivated by these studies, we propose a novel scheme for efficient content-based medical image retrieval that utilizes similarity measures defined over higher-level patterns associated with clusters of low-level image feature spaces. The term pattern is defined in the context of a state of the art framework named PANDA (PAtterns for Next generation Database systems) [27].

The rest of this paper comprises four sections. Section 2 outlines the PANDA framework. The proposed pattern similarity scheme is described in Section 3, and the results obtained from an indicative application for the retrieval of radiographs are apposed in Section 4. Finally, Section 5 summarizes the conclusions and the future perspectives of this study.

\section{THE PANDA FRAMEWORK}

The efficient management of patterns extracted from medical image databases is of vital importance due to the 
extremely big storage requirements as well as the complexity of such kind of raw data. Taking advantage of the PANDA framework [27] we adopt the idea of a Pattern Based Management System (PBMS) as the infrastructure for managing patterns extracted from our CBIR scheme, in contrast to DataBase Management Systems (DBMS). The key concept of PANDA is that any type of pattern can be represented in a compact and unified way. This can be achieved through a Pattern-Base (PB) keeping information about extracted patterns. Such a PB introduced in [27] consists of three basic layers: the pattern, the pattern type and the class. A pattern type is a description of the pattern structure. A pattern is an instance of the corresponding pattern type and class is a collection of semantically related patterns of the same pattern type.

Formally, a pattern type $p t$ is a quintuple $p t=<n$, $s s, d s$, $m s, f>$, where $n$ is the name of the pattern type. The structure schema ss defines the pattern space by describing the structure of the pattern type, while the source schema $d s$ defines the data source space by describing the dataset wherefrom patterns of this pattern type are derived. The measure schema $m s$, quantifies the quality of the source data representation achieved by patterns of this pattern type and the formula $f$ describes the relationship between the source space and the pattern space. In this notation, if $p t$ is a pattern type then $p=\langle p i d, s, d, m, e>$ is an instance of $p t$, where pid is a unique pattern identifier, $s, d, m$ are the corresponding structure, source and measure of the pattern, while $e$ is an expression indicating the section of the source space related to pattern $p$.

Aside from the physical storage of the patterns we appropriately utilize the PANDA framework for the comparison of patterns [27] as the base of our image retrieval methodology in our CBIR scheme. In PANDA the similarity $\operatorname{sim}, \operatorname{sim} \in[0,1]$ between two patterns $p_{1}, p_{2}$ of the same type can be computed by combining, by means of an aggregation function $f_{\text {aggr }}$, the similarity between both the structure $s$ and the measure $m$ components:

$\operatorname{sim}\left(p_{1}, p_{2}\right)=f_{\text {aggr }}\left(\operatorname{sim}_{\text {struct }}\left(p_{1} . s, p_{2} . s\right), \operatorname{sim}_{\text {meas }}\left(p_{1} \cdot m, p_{2} \cdot m\right)\right)(1)$

Efficient definition of the structure and measure of patterns extracted from medical images, as well as appropriate selection of an aggregation logic and distance functions to assess the respective similarities, is one of the challenges adopted in this paper.

\section{MEdicAl IMAgE Retrieval USING PATterns}

The proposed content-based medical image retrieval scheme is outlined in Fig. 1. It involves four steps: a) lowlevel feature extraction from each of the stored and the query images, b) clustering of the extracted feature vectors per image, c) pattern instantiation of the clusters, and d) computation of pattern similarities. The registration of a new image in the database involves the first three of the four steps described for image retrieval (a, b, and c).

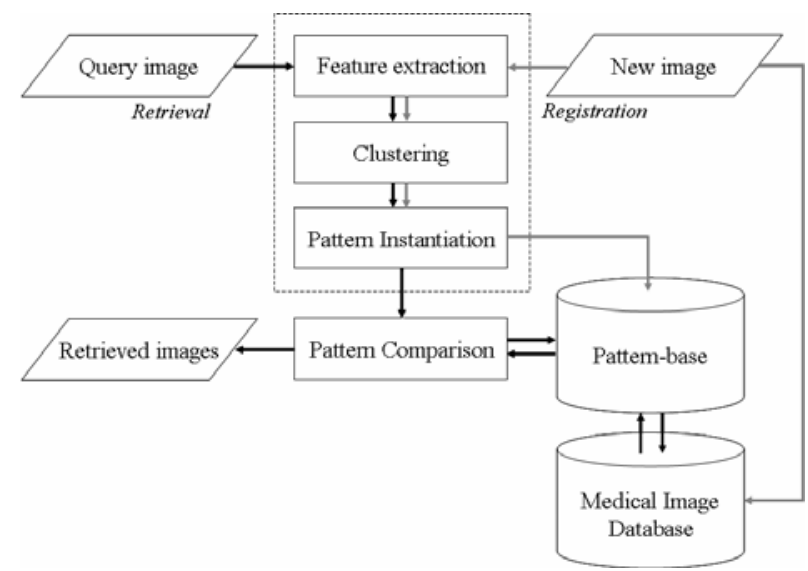

Fig. 1. Outline of the content-based medical image retrieval methodology that embodies the proposed pattern similarity scheme. The black arrows indicate the data flow for image retrieval, whereas the grey arrows indicate the data flow for the registration of a new image.

\section{A. Low-Level Image Feature Extraction}

Each of the images stored in the database, as well as the query image is raster scanned with a sliding window of userdefined size and sliding step. The sliding step may allow windows to overlap between each other. For each window $N$ features $f_{i}, i=1,2, N$ are calculated to form a single feature vector $F$. The number of feature vectors produced for each image depends on the size, the dimensions and the step of the sliding window.

Typically, the features characterizing the image content should be selected based on the details associated with the image collection and the retrieval task [28]. Color, texture and shape are the three major classes of image features used in image retrieval [1][9][28]. However, this paper focuses on the utility of the proposed pattern similarity scheme rather than on the selection of an optimal feature set for a particular image retrieval task.

\section{B. Clustering}

For the clustering of the extracted image features we used the Expectation Minimization (EM) algorithm [37]. The EM algorithm is a widely-used statistical clustering method. It performs clustering by estimating the mean and standard deviation of each cluster, so as to maximize the likelihood of the observed data.

\section{Pattern Instantiation}

Given a clustering of an image comprising $M$ clusters $C_{i}$, $i=1,2, . ., M$, according to the PANDA formalization and with respect to the output of the EM algorithm a pattern specimen $_{i}$ is instantiated for each cluster $C_{i}$ representing a physical anatomic specimen depicted in a medical image:

$$
\text { specimen }_{i}=\left(\begin{array}{l}
S S:\left(D:\left[[\text { mean }:[\text { Real }], \text { stdDev }:[\text { Real }]]_{1}^{\mathrm{N}}\right),\right. \\
M S:(p p: \text { Real })
\end{array}\right)
$$

where mean and stdDev are the mean and the standard deviation of the distribution $D_{\mathrm{j}}$ for every one of the $N$ features $(j=1,2, . ., N)$ in cluster $C_{i}$, respectively, and $p p$ is the 
prior probability of $C_{i}$. Here prior probability is defined as the fraction of the feature vectors of the image that belong to cluster $C_{i}$. Intuitively, prior probability $p p$ is equivalent with the support measure widely used in data mining models. In our case, in addition to the qualitative aspect of the prior probability, it also provides an indication of the size of the specimen.

In this connection, a medical image is considered as a complex pattern defined by (3), consisting of a set of simple clusters each one of them represented by the mean and standard deviation values of a distribution.

$$
\text { medical_image }=\left(\begin{array}{l}
S S:\{\text { Specimen }\} \\
M S: \perp
\end{array}\right)
$$

\section{Computation of Pattern Similarities}

Aiming at the definition of the similarity of two medical images (i.e., complex patterns), we have first to define the measures' and the structural similarity between two clusters $C_{1}$ and $C_{2}$ (i.e., simple patterns). The measures' similarity between two clusters is computed using the Euclidean distance as in Eq.(4)

$$
\operatorname{sim}_{\text {meas }}\left(C_{1}, C_{2}\right)=1-\left|C_{1} \cdot p p-C_{2} \cdot p p\right|
$$

Rephrasing the problem of defining the structural similarity between $C_{1}$ and $C_{2}$ we need to find a measure for evaluating the closeness of two sets of distributions, as $C_{1}$ and $C_{2}$ are. Further decomposing the problem, we should first define a method of computing the similarity between just two distributions $D_{1}$ and $D_{2}$. To achieve this, we extend the methodology proposed in [38] where two distributions can be compared by discovering the expected percentage the first distribution overlaps the other. This is achieved by consulting a nomogram and more specifically by interpolating between contours of the nomogram to estimate the expected percentage. The previously mentioned nomogram is constructed by taking as $x$ axis a Standardized Absolute Distance between the means of the distributions ( $S A D M$, Eq. 5), whereas the $y$ axis is computed as the Ratio of the Standard Deviations of the distributions (RSD, Eq. 6).

$$
\begin{gathered}
S A D M=\frac{\mid D_{1} \cdot \text { mean }-D_{2} \cdot \text { mean } \mid}{D_{1} \cdot \text { stdDev }} \\
R S D=\frac{D_{2} \cdot s t d D e v}{D_{1} \cdot s t d D e v}, \text { where } D_{1} \cdot \text { stdDev }<D_{2} \cdot \text { stdDev }
\end{gathered}
$$

Given the above, we define the similarity between distributions $D_{1}$ and $D_{2}$, by Eq.(7).

$$
\operatorname{sim}\left(D_{1}, D_{2}\right)=\left\{\begin{array}{l}
1-\frac{\mid D_{1} \cdot \text { mean }-D_{2} \cdot \text { mean } \mid \cdot D_{1} \cdot s t d D e v}{D_{1} \cdot s t d D e v^{2}}, \\
\text { if } \mid D_{1} \cdot \text { mean }-D_{2} \cdot \text { mean } \mid \cdot D_{1} \cdot s t d D e v<D_{1} \cdot s t d D^{2} v^{2} \\
0, \text { otherwise }
\end{array}\right.
$$

This is a means to automate and materialize the intuitive overlap between two distributions. In other words, the similarity is defined by finding the proximity of the mean values, normalized by the standard deviation of the first and by weighting with a factor indicating how bigger the standard deviation of the second in contrast to the first is.
Having this, we let the structural similarity between two sets of distributions (i.e. two clusters $C_{1}$ and $C_{2}$ ) be the average among the similarities computed for each pair of the $N$ features:

$$
\operatorname{sim}_{\text {struct }}\left(C_{1}, C_{2}\right)=\sum_{j=1}^{N} \operatorname{sim}\left(D_{j}^{1}, D_{j}^{2}\right) / N
$$

We aggregate the similarities between the qualitative $\operatorname{sim}_{\text {meas }}$ and the structural $\operatorname{sim}_{\text {struct }}$ similarities between the clusters by using the following aggregation function $f_{\text {aggr }}$, which gives the same weight to either of the above similarities, while further weights the overall similarity by the prior probabilities of the clusters, as a bias towards similar and concurrently big clusters.

$$
\operatorname{sim}\left(C_{1}, C_{2}\right)=\frac{\operatorname{sim}_{\text {struct }}\left(C_{1}, C_{2}\right)+\operatorname{sim}_{\text {meas }}\left(C_{1}, C_{2}\right)}{2} \cdot C_{1} \cdot p p \cdot C_{2} \cdot p p
$$

Having defined the similarity between clusters (i.e., simple patterns), to compare two medical images $M I_{1}$ and $M I_{2}$ (i.e., complex patterns) we need to determine the coupling methodology between the different clusters of each image. Though various coupling types can be applied in the context of the PANDA framework [27], we adopt the matching of Eq.(10), allowing each cluster of the first image to match more than one cluster of the second, and vice versa.

$$
\operatorname{sim}\left(M I_{1}, M I_{2}\right)=\frac{1}{M^{2}} \cdot \sum_{i=1}^{M} \sum_{j=1}^{M} \operatorname{sim}\left(C_{i}^{M I_{1}}, C_{j}^{M I_{2}}\right)
$$

\section{Results}

Experiments were performed with radiographic images from the IRMA (Image Retrieval in Medical Applications) dataset [29]. This is a growing collection of radiographic images acquired in RWTH Aachen University of Technology Hospital, Germany. It is used as reference for medical image retrieval tasks. It currently contains 10,000 arbitrarily selected anonymous radiographic images for which the ground truth information is provided. The radiographs span 116 categories and depict various anatomic specimens, including cranium, spine, arm, chest, abdomen, leg, pelvis, breast, and hands of patients of various ages, genders, and pathologies. All radiographic images are in 8bit greyscale format and have been downscaled to fit into a $512 \times 512$ bounding box maintaining the original aspect ratio.

The aim of the experiments was to demonstrate the efficiency of the proposed pattern similarity scheme for CBIR over the conventional scheme used by CBIR systems. To evaluate its performance a subset of $90 \%$ of the images was registered in the database, whereas a subset of $10 \%$ of the images was used for querying the pattern-base. Each image was sampled using overlapping windows of $32 \times 32$ pixel dimensions with an 8-pixel sliding step, resulting in a total of 625 samples.

From each sample we considered the extraction of a standard, yet effective set of features comprising 3-level Discrete Wavelet Transform (DWT) energy features 
[10][31]. The dimension of each feature vector is $N=10$.

The feature vectors extracted from each image were clustered by means of the EM algorithm implemented in the WEKA data mining tool [36].

In order to determine the optimal number of distinctively discriminated clusters per image, we followed a visualization approach to representation and understanding of the spatial relationships between the categories of the image content. This was achieved by means of geometric projection techniques [31] and the three-dimensional classpreserving projection algorithm [32]. During the projection procedures, class-preserving projection techniques preserve the properties of the clustered data in the $R^{n}$ space also to the projection plane in order to construct corresponding representations from which accurate inferences could be extracted. The results from the application of this visualization method on a representative image from each anatomic specimen showed that the optimal number of distinctive clusters per image is $M=4$. An example threedimensional projection of the clustered feature space of an image is illustrated in Fig. 2.

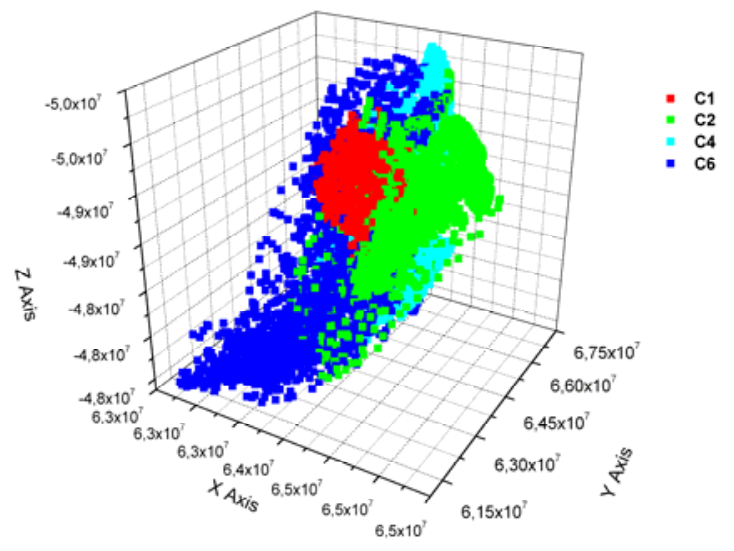

Fig. 2. Three-dimensional projection of the feature space of a radiographic image clustered into four categories by the EM algorithm.

For each cluster a pattern specimen $_{i}, i=1,2,3,4$ (Eq.2) was assigned, whereas each image was represented by a complex pattern medical_image (Eq.3). The collection of patterns originating from the images registered in the database was used to build the pattern-base. Subsequent queries were executed to evaluate the performance of the proposed scheme.

An indicative example retrieval of five radiographic images, based on the content of a query image is illustrated in Fig. 3. It can be noticed that the retrieved images contain semantically relevant regions (spine) although they belong to different categories. This could be justified if one considers that the proposed CBIR scheme involves features extracted locally from the images. Similar observations are valid for queries performed using radiographic images from other categories, and indicate that the patterns used for image representation carry substantial semantic information.

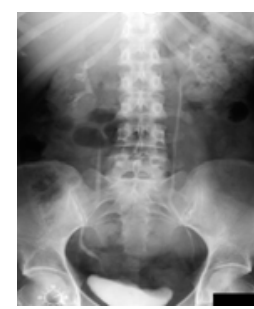

(a)

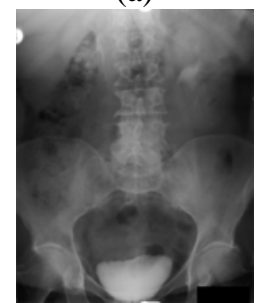

(d)

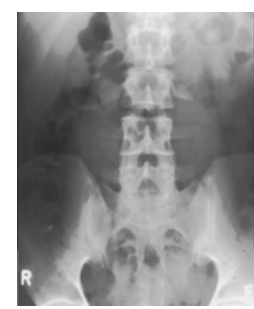

(b)

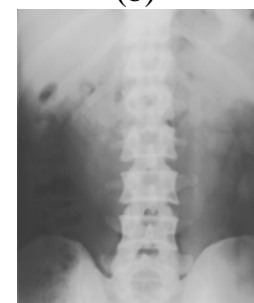

(e)

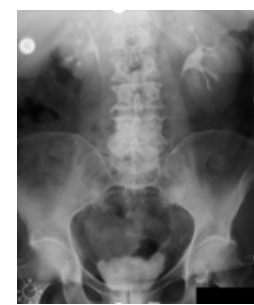

(c)

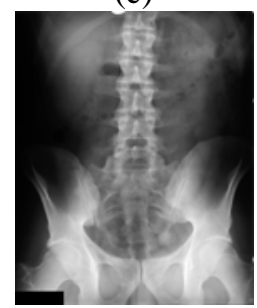

(f)
Fig. 3. Indicative example retrieval of radiographic images: (a) Query image (category: abdomen, uropoietic system), (b)-(d) Correct retrievals, (e)-(f) False retrievals (category: abdomen, gastrointestinal system).

Figure 4 illustrates the performance of the proposed pattern similarity scheme that involves pattern comparisons, as compared with the performance of the conventional scheme that involves exhaustive comparisons of the feature vectors. The performance is measured in terms of the logarithm of the number of comparisons between the query and the registered data. The results show that the proposed scheme achieves approx. 2,400 less comparisons.

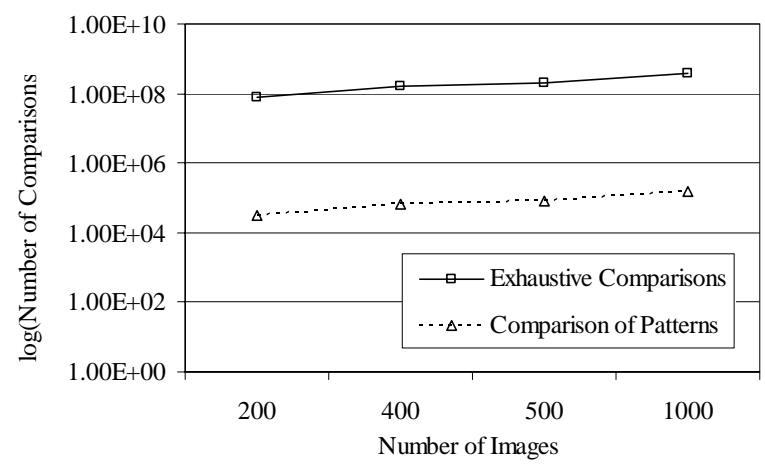

Fig. 4. Number of comparisons between the query and the registered data for the conventional and the proposed scheme.

\section{Conclusions}

We presented a novel scheme for efficient content-based medical image retrieval. This scheme utilizes patterns, defined in the context of the state of the art framework PANDA. Image retrieval is based on similarities defined over complex patterns. Searching for similar images involves comparison of patterns instead of exhaustive, nearest neighbor searching over the whole set of the feature vectors stored in the image database. The results advocate to the efficiency of the proposed scheme for image retrieval from large databases over the commonly employed methods.

The retrieval performance of a content-based medical 
image retrieval system implementing the proposed scheme is highly associated with the feature set used for data representation. Selection of representative features, can lead to separable clusters, and provided that the clustering method chosen is compatible with the geometry of the feature space used, it is possible to minimize the performance divergence of the proposed and the conventional schemes.

By storing clustering patterns along with the low-level features set in a unified format we facilitate further processing and analysis. To date, most data mining algorithms have concentrated on the extraction of interesting rules directly from low-level data [33]. Our approach provides the means for deriving rules from the results of other data mining algorithms that is, mining from rules set. In the current work our initial low-level feature set is further processed and represented via clustering by higher-level patterns which are in a machine processible format. A significant advantage of this approach is that the nature of the rules to be extracted by this process contains different higher order semantics.

Future perspectives of this work include: a) the systematic evaluation of the proposed scheme for the retrieval of various medical images, such as endoscopic [34] and ultrasound images [35] according to their pathology, b) the enhancement of the retrieval performance by using image indexing techniques based on specialized data structures [19]-[22], c) the integration of the proposed scheme with ontology-based information extraction and data mining techniques for the retrieval of medical images using heterogeneous data sources.

\section{ACKNOWLEDGMENT}

The collection of images used in this study is courtesy of T.M. Lehmann, Image Retrieval in Medical Application (IRMA) group, Dept. of Medical Informatics, RWTH Aachen, Germany, http://irma-project.org.

\section{REFERENCES}

[1] C. Faloutsos, R. Barber, M. Flickner, J. Hafner, W. Niblack, D. Petkovic, and W. Equitz, "Efficient and Effective Querying by Image Content," Journal of Intelligent Information Systems, vol. 3, pp. 231262, 1994.

[2] J.R. Smith, and S.F. Chang, "Visualseek: A Fully Automated ContentBased Image Query System," in Proc. 1996 ACM Int'l Multimedia Conf., pp. 87-98, 1996.

[3] A. Hampapur, A. Gupta, B. Horowitz, C. F. Shu, C. Fuller, J. Bach, M. Gorkani, and R. Jain, "Virage video engine," in Proc. SPIE: Storage and Retrieval for Image and Video Databases V, pp. 188-197, San Jose, February 1997.

[4] W.Y. Ma, and B.S. Manjunath, "Netra: A Toolbox for Navigating Large Image Databases,” Multimedia System, vol. 7, pp. 184-198, 1999.

[5] J. Laaksonen, M. Koskela, S. Laakso, and E. Oja, "PicSOM - ContentBased Image Retrieval with Self-Organizing Maps," Pattern Recognition Letters, vol. 21, pp. 1199-1207, 2000.

[6] J.Z. Wang, J. Li, and G. Wiederhold, "SIMPLIcity: SemanticsSensitive Integrated Matching for Picture Libraries," IEEE Trans.
Pattern Analysis and Machine Intelligence, vol. 23, no. 9, pp. 947963, Sept. 2001.

[7] Q. Iqbal, and J. K. Aggarwal, "CIRES: A System for Content-based Retrieval in Digital Image Libraries," in Proc. International Conference on Control, Automation, Robotics and Vision (ICARCV), Singapore, pp. 205-210, December 2-5, 2002.

[8] T. Deselaers, D. Keysers, and H. Ney, "FIRE - Flexible Image Retrieval Engine: ImageCLEF 2004 Evaluation,”, LNCS 3491, pp. 688-698, 2004.

[9] R. Veltcamp, and M. Tanase, "Content--Based Image Retrieval Systems: A Survey,” Tech. Report UU-CS-2000-34, Dept. of Computing Science, Utrecht Univ., 2000.

[10] H. Müller, N. Michoux, D. Bandon, A. Geissbuhler, "A Review of Content-Based Image Retrieval Systems in Medicine - Clinical Benefits and Future Directions," International Journal of Medical Informatics, vol. 73, pp. 1-23, 2004.

[11] C. R. Shyu, C. E. Brodley, A. C. Kak, A. Kosaka, A. M. Aisen, and L. S. Broderick, "ASSERT: A Physician-in-the-loop Content-Based Image Retrieval System for HRCT Image Databases," Computer Vision and Image Understanding, pp. 111-131, 1999.

[12] F. Schnorrenberg, C. S. Pattichis, C. N. Schizas, K. Kyriacou, "Content-Based Retrieval of Breast Cancer Biopsy Slides," Technology and Health Care, vol. 8, pp. 291-297, 2000.

[13] W. Cai, D. D. Feng, R. Fulton, "Content Based Retrieval of Dynamic PET Functional Images," IEEE Trans. Information Technology in Biomedicine, vol. 4, no. 2, pp. 152-158, 2000.

[14] D.-M. Kwak, B.-S. Kim, O.-K. Yoon, C.-H. Park, J.-U. Won, K.-H. Park, "Content-Based Ultrasound Image Retrieval Using a Coarse to Fine Approach," Annals of the New York Academy of Sciences, vol. 980, pp.212-224, 2002.

[15] S. Chen, and L. Li, "Build a Content Based Image Retrieval System of Endoscopic Image," in Proc. Medical Informatics Symposium (MIST 03), Taiwan, Sept. 2003.

[16] Lei Zheng, A.W. Wetzel, J. Gilbertson, M.J. Becich, "Design and Analysis of a Content-Based Pathology Image Retrieval System," IEEE Trans. Information Technology in Biomedicine, vol.7, no.4, pp. 249- 255, Dec. 2003.

[17] S. Antani, X. Xu, L.R. Long, G.R. Thoma, "Partial Shape Matching for CBIR of Spine X-ray Images," in Proc IS\&T/SPIE Electronic Imaging - Storage and Retrieval Methods and Applications for Multimedia, SPIE vol. 5307, pp. 1-8, 2004.

[18] I. El-Naqa, Y. Yang, N.P. Galatsanos, R.M. Nishikawa, M.N. Wernick, "A similarity learning approach to content-based image retrieval: application to digital mammography,” IEEE Trans. Med Imaging, vol. 23, no. 10, pp. 1233-1244, Oct. 2004.

[19] E. G.M. Petrakis, C. Faloutsos, "Similarity Searching in Medical Image Databases," IEEE Trans. Knowledge and Data Engineering, vol. 9, no. 3, pp. 435-447, May-June, 1997.

[20] W.I. Grosky and R. Mehrotra, "Indexed-Based Object Recognition in Pictorial Data Management," Computer Vision, Graphics, and Image Processing, vol. 52, pp. 416-436, 1990.

[21] J.K. Wu, and A.D. Narasimhalu, "Identifying Faces Using Multiple Retrievals,” IEEE Multimedia, vol. 1, no. 2, pp. 20-38, 1994.

[22] W.-M. Jeng, and J.-H. Hsiao, "An Efficient Content Based Image Retrieval System Using the Mesh-of-Trees Architecture," J. Information Science and Engineering, vol. 21, pp. 797-808, 2005.

[23] R.O. Stehling, A.X. Falcao, and M.A. Nascimento, "An Adaptive and Efficient Clustering-Based Approach for Content-Based Image Retrieval in Image Databases," in Proc. International Symposium on Database Engineering \& Applications (IDEAS 01), pp. 56-365, 2001.

[24] R. Zhang, Z.(M) Zhang, "A Clustering Based Approach to Efficient Image Retrieval" in Proc. 14th IEEE International Conference on Tools with Artificial Intelligence (ICTAI’02), p. 339, 2002.

[25] A. Berman, and L. G. Shapiro, "Efficient Image Retrieval with Multiple Distance Measures," in Proc. SPIE Conference on Storage and Retrieval for Image and Video Databases, pp. 12-21, 1997.

[26] R.O. Stehling, M.A. Nascimento, and A.X. Falcão, “A Compact and Efficient Image Retrieval Approach based on Border/Interior Pixel Classification," in Proc. $11^{\text {th }}$ Int. Conference on Information and Knowledge Management (CIKM`02), ACM Press, NY, pp. 102-109.

[27] I. Bartolini, P. Ciaccia, I. Ntoutsi, M. Patella, and Y. Theodoridis, "A Unified and Flexible Framework for Comparing Simple and Complex 
Patterns”, in Proc. 8th European Conference on Principles and Practice of Knowledge Discovery in Database, pp. 496-499, 2004.

[28] T. Deselaers, D. Keysers, H. Ney, "Features for Image Retrieval-A Quantitative Comparison,” in Proc. 26th DAGM Symposium, LNCS, pp. 228-236, 2004.

[29] T.M. Lehmann, M.O. Güld, C. Thies, B. Plodowski, D. Keysers, B. Ott, H. Schubert, "IRMA - Content-based image retrieval in medical applications,” in Proc. 14th World Congress on Medical Informatics (Medinfo 2004), IOS Press, Amsterdam, vol. 2, pp. 842-848, 2004.

[30] J.Z. Wang, G. Wiederhold, O. Firschein, and S.X. Wei, "ContentBased Image Indexing and Searching Using Daubechies' Wavelets,” Int. Journal of Digital Libraries, vol. 1, no. 4, pp. 311-328, 1998.

[31] I.S. Dhillon, D.S. Modha, W.S. Spangler, "Class Visualization of High-Dimensional Data with Application,” IBM Almaden Research Center, San Jose, 1999.

[32] I. Kopanakis, and B. Theodoulidis, "Visual Data Mining Modelling Techniques for The Visualization of Mining Outcomes," Journal of Visual Languages and Computing, Special Issue on Visual Data Mining, vol. 14, no.6, pp. 543-589, 2003.
[33] M. Spiliopoulou, and J. F. Roddick "Higher Order Mining: Modellingand Mining the Results of Knowledge Discovery", in Proc. International conference on data mining, vol. 2, pp. 309-320, 2000.

[34] S.A. Karkanis, D.K. Iakovidis, D.E. Maroulis, D.A. Karras, and M. Tzivras, "Computer Aided Tumor Detection in Endoscopic Video using Color Wavelet Features,” IEEE Trans. Information Technology in Biomedicine, vol. 7, pp. 141-152, 2003.

[35] D.E. Maroulis, M. Savelonas, S.A. Karkanis, D.K. Iakovidis, and N. Dimitropoulos, "Computer-Aided Thyroid Nodule Detection in Ultrasound Images," in Proc. IEEE International Symposium on Computer-Based Medical Systems (CBMS), Dublin, Ireland, pp. 271276, 2005.

[36] I. H. Witten, and E. Frank, "Data Mining: Practical machine learning tools and techniques”, 2nd Edition, Morgan Kaufmann, San Francisco, 2005.

[37] A. Dempster, N. Laird, and D. Rubin. "Maximum likelihood from incomplete data via the EM algorithm," Journal of the Royal Statistical Society, Series B, vol. 39, no.1, pp.1-38, 1977.

[38] J.M. Linacre, “Overlapping Normal Distributions,” Rasch Measurement Transactions, vol. 10, no. 1 pp. 487-488, 1996. 